\title{
Management of Patients with Primary Hyperparathyroidism and Concurrent Thyroid Disease: An Evolving Field
}

\author{
Philip M. Spanheimer, MD and Ronald J. Weigel, MD, PhD \\ Department of Surgery, University of Iowa, Iowa City, IA
}

The presence of thyroid disease can complicate the evaluation and surgical management of patients with primary hyperparathyroidism (PHPT). Retrospective analysis has shown that approximately $20-50 \%$ of patients with PHPT also have thyroid disease. ${ }^{1-3}$ The reported incidence of thyroid malignancy in patients with PHPT is approximately $3-4 \% .^{1,4}$ There is no uniformly accepted protocol for the evaluation of patients with thyroid pathology before operative intervention for PHPT. Neck exploration for PHPT normally provides excellent access to the thyroid, whereas minimally invasive parathyroidectomy offers a more limited exposure of the thyroid gland. The increased application of the minimally invasive approach has increased the reliance on preoperative imaging. Modalities for localizing abnormal parathyroid glands such as ${ }^{99 \mathrm{~m}} \mathrm{Tc}-$ sestamibi scintigraphy (Tc-MIBI), I-123, and neck ultrasound can also identify thyroid pathology.

Onkendi et al. analyzed the results from a series of patients with PHPT and concurrent thyroid nodules who underwent dual-isotope Tc-MIBI and radioiodine (I-123) subtraction imaging. ${ }^{5}$ The most significant finding is that the phenotype Tc-MIBI-Hot/I-123-Cold has a negative predictive value for malignancy of $90 \%$. Patients with the Hot/Cold phenotype were almost 3 times as likely to have a malignant thyroid lesion, indicating that these patients should be evaluated more aggressively with a lower threshold for fine-needle aspiration biopsy (FNAB) and thyroid resection. Patients with thyroid nodules that had

(C) Society of Surgical Oncology 2012

Published Online: 7 March 2012

R. J. Weigel, MD, PhD

e-mail: Ronald-Weigel@uiowa.edu any other phenotype (Hot/Hot, Cold/Hot, Cold/Cold) had a much lower potential for thyroid malignancy, and therefore, it may be appropriate to treat these patients with close follow-up rather than FNAB or thyroid resection.

Onkendi et al. further identified a group of patients who were found during surgery to have a false-positive imaging result, characterized by a Hot/Cold scan with no abnormal parathyroid found corresponding to the imaging localization. Patients with underlying thyroid malignancy were more likely to have a false-positive parathyroid scintigraphy (45\% compared to $22 \%$ of patients with benign thyroid disease). These data indicate that patients undergoing neck exploration who are found to have a false-positive dual imaging result should have further intraoperative evaluation for the potential of an undiagnosed thyroid malignancy.

Management of patients with PHPT and concurrent thyroid disease is an evolving field. Onkendi et al. provide useful information to identify patients requiring further assessment of concurrent thyroid nodules. However, because of the significant cost and logistics of using the I-123 isotope, many imaging protocols for PHPT do not utilize I-123 subtraction. For example, other protocols for parathyroid localization include the use of MIBI-pertechnetate subtraction scintigraphy. High-resolution ultrasound is also commonly used to localize abnormal parathyroid glands and is particularly valuable for identifying thyroid nodules. Both MIBI-pertechnetate scintigraphy and neck ultrasound can identify concurrent thyroid nodules requiring further evaluation with FNAB, and these other imaging modalities have the potential to identify a concurrent thyroid malignancy. ${ }^{1,6}$ Interpretation of parathyroid imaging tests can be used to stratify patients with PHPT and thyroid disease according to risk for thyroid malignancy. Further studies are warranted to more clearly define how various parathyroid localization techniques should be interpreted with regard to concurrent thyroid pathology. 


\section{REFERENCES}

1. Kairys JC, Daskalakis C, Weigel RJ. Surgeon-performed ultrasound for preoperative localization of abnormal parathyroid glands in patients with primary hyperparathyroidism. World J Surg. 2006;30:1658-63.

2. Adler JT, Chen H, Schaefer S, Sippel RS. Does routine use of ultrasound result in additional thyroid procedures in patients with primary hyperparathyroidism? J Am Coll Surg. 2010;211:536-9.

3. Heizmann O, Viehl CT, Schmid R, et al. Impact of concomitant thyroid pathology on preoperative workup for primary hyperparathyroidism. Eur J Med Res. 2009;14:37-41.
4. Beus KS, Stack BC. Synchronous thyroid pathology in patients presenting with primary hyperparathyroidism. Am J Otolaryngol. 2004;25:308-312.

5. Onkendi EO, Richards ML, Thompson GB, Farley DR, Peller PJ, Grant CS. Thyroid cancer detection with dual-isotope parathyroid scintigraphy in primary hyperparathyroidism. Ann Surg Oncol. 2012. doi:10.1245/s10434-012-2282-x.

6. Rubello D, Taniato A, Pelizzo MR, Casara D. Papillary thyroid carcinoma associated with parathyroid adenoma detected by pertechnetate-MIBI subtraction scintigraphy. Clin Nucl Med. 2000;25:898-900. 